\title{
Synthesis of Novel Heterocyclic Structures via Reaction of Isocyanides with $S$-trans-enones
}

\author{
Jeffrey D. Winkler* and Sylvie M. Asselin \\ Department of Chemistry, University of Pennsylvania, Philadelphia, PA 19104, U.S.A. \\ winkler@sas.upenn.edu
}

\section{Supplementary Information Table of Contents}

General Methods ............................................................................................. 2

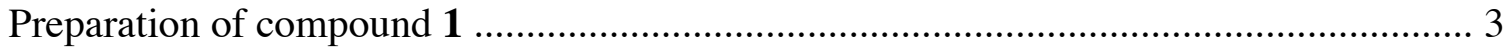

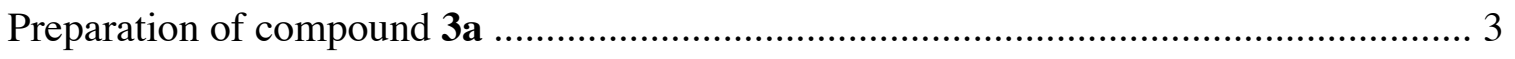

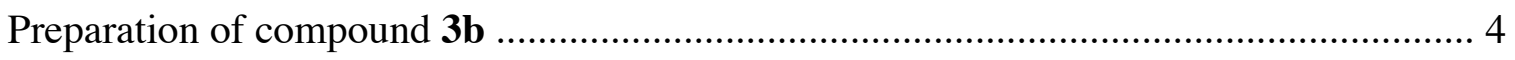

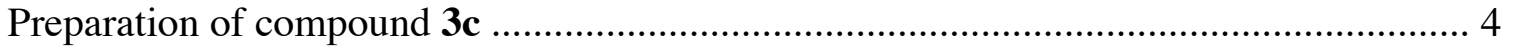

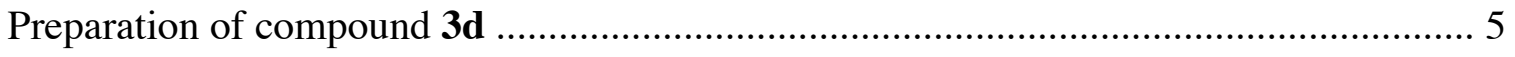

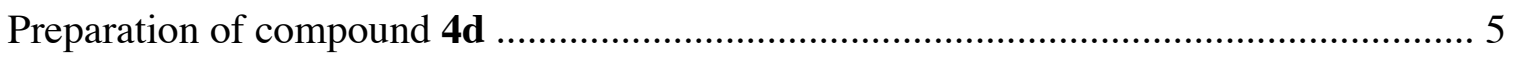

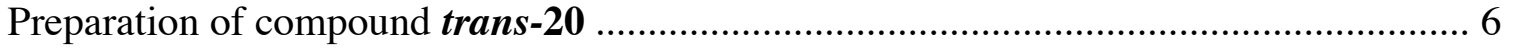

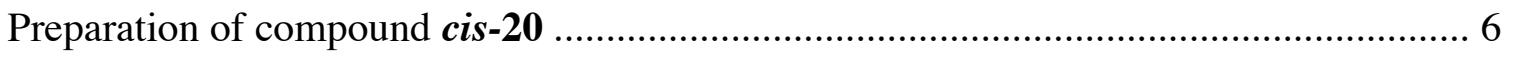

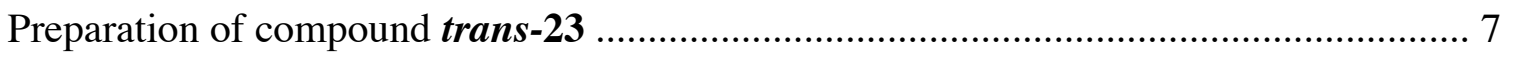

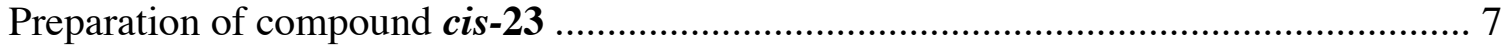

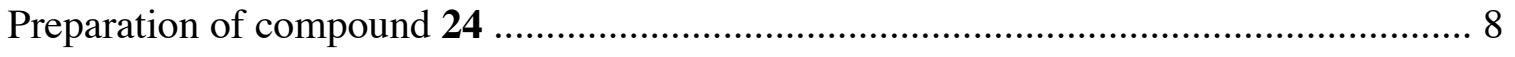

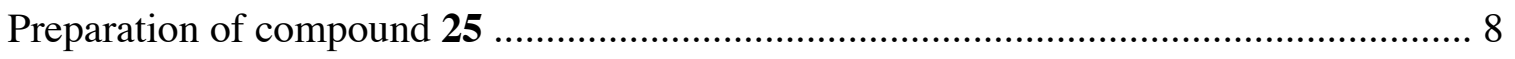

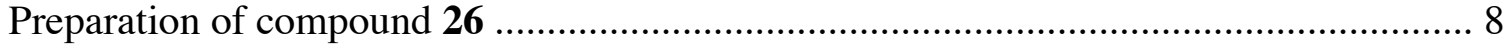

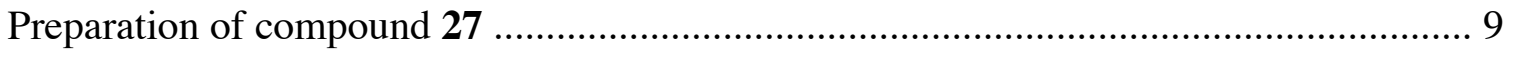

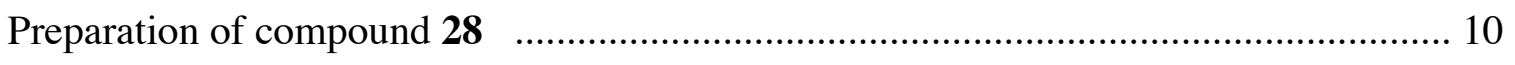

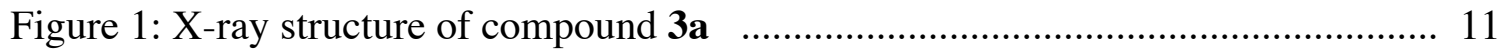

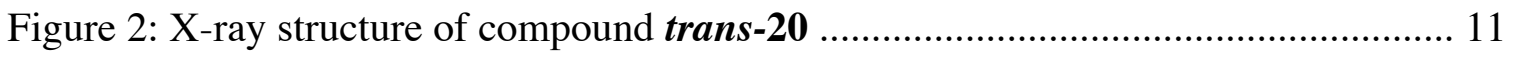

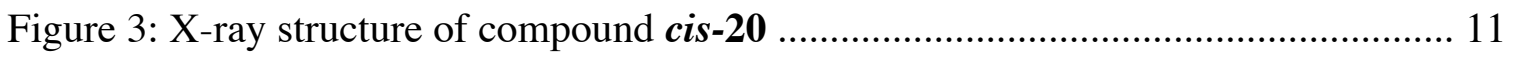

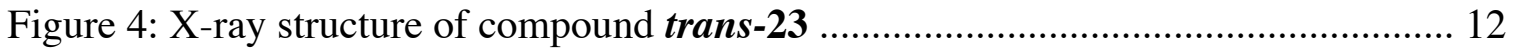

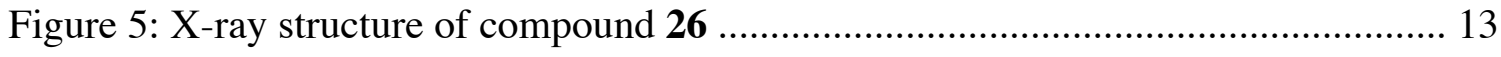




\section{EXPERIMENTAL PROCEDURES}

\section{General Methods}

All reactions were performed in oven- or flame-dried glassware under an argon atmosphere. Solvents used for the reactions (tetrahydrofuran and toluene) were passed through an activated alumina column. ${ }^{1}$ Methylcyclohexane was distilled from calcium hydride. Except as otherwise noted, all other solvents and reagents were A. C. S.-, reagent-, or HPLC-grade (or better) and were used as received from commercial sources (Acros, Aldrich, Fluka, Fisher, TCI, or Strem) or were prepared according to literature procedures. Diethylaluminum chloride was purchased from Aldrich in a blue cylinder as a $1.0 \mathrm{M}$ solution in hexanes. The gallium (III) chloride solution was either purchased from Aldrich as a $0.5 \mathrm{M}$ solution in pentane or prepared from solid gallium (III) chloride $(5 \mathrm{~g}$, Aldrich) and dissolved in methylcyclohexane $(90 \mathrm{~mL})$ to afford a $0.316 \mathrm{M}$ solution and stored in a sure-seal bottle.

Merck precoated silica gel plates $(250 \mu \mathrm{m}, \mathrm{K} 6 \mathrm{~F})$ were used for analytical TLC. The plates were visualized using $254 \mathrm{~nm}$ ultraviolet light and/or by treating the plates with potassium permanganate, ninhydrin, or anisaldehyde stains. Flash column chromatography was performed using silica gel (partical size 32-63 microns) purchased from Sorbent Technologies. Infrared spectra were acquired on a Perkin-Elmer 1600 series Fourier-transform spectrometer using the neat material on $\mathrm{NaCl}$ or $\mathrm{KBr}$ plates. Melting points were determined on a Thomas-Hoover Uni-Melt capillary melting point apparatus and are uncorrected. Bruker AM-500 or DRX-500 instruments were used to acquire ${ }^{1} \mathrm{H}$ and ${ }^{13} \mathrm{C}$ NMR spectra at $500 \mathrm{MHz}$ and $125 \mathrm{MHz}$, repectively. The residual solvent peak of $\mathrm{CDCl}_{3}$, unless otherwise noted, was used as the internal reference $\left(\delta 7.26\right.$ for ${ }^{1} \mathrm{H}$, and $\delta$ 77.0 for ${ }^{13} \mathrm{C}$ ). High-resolution mass spectra were recorded by Dr. Rakesh K. Kohli at the University of Pennsylvania Mass Spectrometry Service Center in positive ion mode using either chemical or electrospray ionization. X-ray crystallographic analyses were performed by Dr. Patrick J. Carroll at the University of Pennsylvania.

$\begin{array}{ll}\text { Abbreviations: } & \\ \text { EA = ethyl acetate } & \mathrm{s}=\text { singlet } \\ \mathrm{HX}=\text { hexanes } & \mathrm{d}=\text { doublet } \\ \text { DCM = dichloromethane } & \mathrm{t}=\text { triplet } \\ \text { EE = diethyl ether } & \mathrm{q}=\text { quartet } \\ \text { BRSM = based on recovered starting material } & \text { quint = quintet } \\ & \mathrm{m}=\text { multiplet } \\ & \mathrm{br}=\text { broad } \\ & \text { app = apparent }\end{array}$

\footnotetext{
${ }^{1}$ Pangborn, A. B.; Giardello, M. A.; Grubbs, R. H.; Rosen, R. K.; Timmers, F. J. Organometallics 1996, 15, 1518.
} 


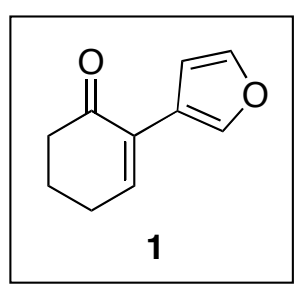

2-Furan-3-yl-cyclohex-2-enone 1: To a solution of 2-bromocyclohex-2-enone ${ }^{2}(1.42 \mathrm{~g}, 8.12 \mathrm{mmol})$ and tetrakis(triphenylphosphine)palladium (0) (Strem, $938 \mathrm{mg}, 0.812 \mathrm{mmol}$ ) in toluene (80 $\mathrm{mL}$ ) was added $2 \mathrm{M} \mathrm{Na}_{2} \mathrm{CO}_{3}(8.1 \mathrm{~mL}, 16.24 \mathrm{mmol})$ and a solution of 3-furylboronic acid (Aldrich, $1.00 \mathrm{~g}, 8.94 \mathrm{mmol}$ ) in absolute ethanol $(8.1 \mathrm{~mL})$. The reaction mixture was heated to $100^{\circ} \mathrm{C}$ for 7.5 hours and then concentrated in vacuo. The resulting residue was partitioned between water and methylene chloride. The aqueous layer was extracted with methylene chloride and the combined organic layers were washed with brine, dried over sodium sulfate, filtered and concentrated.

Purification by silica gel chromatography (5\% EA/HX to $10 \% \mathrm{EA} / \mathrm{HX}$ ) afforded 2furan-3-yl-cyclohex-2-enone 1 ( $847 \mathrm{mg}$, $64 \%)$ as a pale yellow liquid.

$\mathrm{R}_{f}=0.40$ (25\% EA/HX); IR (film, $\mathrm{cm}^{-1}$ ): 2926, 1675, 1159, 1037, 872, 791; ${ }^{1} \mathrm{H}$ NMR $\left(500 \mathrm{MHz}, \mathrm{CDCl}_{3}\right) \delta: 8.05(\mathrm{~s}, 1 \mathrm{H}), 7.36($ app t, J $=1.6 \mathrm{~Hz}, 1 \mathrm{H}), 7.12(\mathrm{t}, \mathrm{J}=4.5 \mathrm{~Hz}, 1 \mathrm{H})$, $6.51(\mathrm{dd}, \mathrm{J}=0.6 \mathrm{~Hz}, \mathrm{~J}=1.8 \mathrm{~Hz}, 1 \mathrm{H}), 2.55-2.49(\mathrm{~m}, 4 \mathrm{H}), 2.06$ (app quint, $\mathrm{J}=6.3 \mathrm{~Hz}, 2 \mathrm{H}$ ); ${ }^{13} \mathrm{C}$ NMR $\left(125 \mathrm{MHz}, \mathrm{CDCl}_{3}\right)$ 8: 197.6, 144.4, 142.2, 131.4, 119.8, 107.9, 39.0, 26.3, 22.7; HRMS (CI) Calculated for $\mathrm{C}_{10} \mathrm{H}_{10} \mathrm{O}_{2}(\mathrm{M}+)$ : 162.0681, found: 162.0677 .

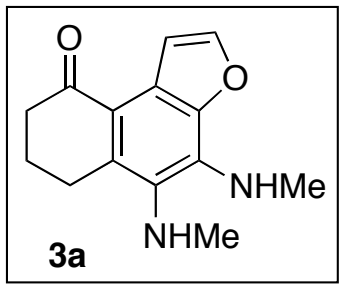

Benzofuran 3a: To a flask containing 2-furan-3-yl-cyclohex-2enone 1 (64 $\mathrm{mg}, 0.395 \mathrm{mmol}$ ) was added methylisocyanide ${ }^{3}$ from a $0.183 \mathrm{M}$ stock solution in THF $(1.94 \mathrm{~mL}, 0.356 \mathrm{mmol})$. Molecular sieves were added and the reaction mixture was cooled to $0^{\circ} \mathrm{C}$. Diethylaluminum chloride $(1.0 \mathrm{M}$ solution in hexanes, $0.43 \mathrm{~mL}, 0.435 \mathrm{mmol}$ ) was added and the reaction was allowed to reach room temperature and stir at room temperature for 18 hours. The reaction mixture was then poured into an aqueous potassium carbonate solution (200 $\mathrm{mg} \mathrm{K} \mathrm{CO}_{3}$ in $10 \mathrm{~mL} \mathrm{H}_{2} \mathrm{O}$ ) and extracted with ethyl acetate. The combined organic layers were dried over sodium sulfate, filtered and concentrated. Purification by silica gel chromatography (0\% - 6\% EE/DCM) afforded benzofuran 3a (15.9 mg, 37\%, 64\% BRSM) as a yellow crystalline solid.

$\mathrm{X}$-ray Structure shown on page 11.

m.p. $=97-98{ }^{\circ} \mathrm{C} ; \mathrm{R}_{f}=0.23(6 \% \mathrm{EE} / \mathrm{DCM})$ and $\mathrm{R}_{f}=0.33(50 \% \mathrm{EA} / \mathrm{HX}) ; \mathrm{IR}\left(\right.$ film, $\left.\mathrm{cm}^{-1}\right)$ : 3354, 2938, 2869, 1646, 1602, 1570, 1534, 1388, 1306, 1185, 1133; ${ }^{1} \mathrm{H}$ NMR $(500 \mathrm{MHz}$, $\left.\mathrm{CDCl}_{3}\right)$ \&: $7.68(\mathrm{~d}, \mathrm{~J}=2.0,1 \mathrm{H}), 7.64(\mathrm{~d}, \mathrm{~J}=2.0 \mathrm{~Hz}, 1 \mathrm{H}), 5.45(\mathrm{br} \mathrm{s}, 1 \mathrm{H}, \mathrm{NH}), 3.40(\mathrm{~s}$, $3 \mathrm{H}$ ), $2.89(\mathrm{t}, \mathrm{J}=6.0 \mathrm{~Hz}, 2 \mathrm{H}), 2.62(\mathrm{~s}, 3 \mathrm{H}), 2.59(\mathrm{t}, \mathrm{J}=6.2 \mathrm{~Hz}, 2 \mathrm{H}$ ), 2.14 (quint, $\mathrm{J}=6.2$ $\mathrm{Hz}, 2 \mathrm{H}$ ), 1.9-1.5 (br s, $1 \mathrm{H}, \mathrm{NH}$ ); ${ }^{13} \mathrm{C}$ NMR (125 MHz, $\mathrm{CDCl}_{3}$ ) $\delta: 196.9,146.2,142.1$, 137.2 (2 carbons accidentally equivalent), 127.0, 126.7, 114.0, 108.5, 38.9, 34.9, 32.4,

\footnotetext{
${ }^{2}$ Smith III, A. B.; Branca, S. J.; Pilla, N. N.; Guaciaro, M. A. J. Org. Chem. 1982, 47(10), 1855-1869.

${ }^{3}$ Cappon, J. J.; Witters, K. D.; Baart, J.; Verdegem, P. J. E.; Hoek, A. C.; Luiten, R. J. H.; Raap, J.; Lugtenburg, J. Recl. Trav. Chim. Pays-Bas 1994, 113, 318-328.
} 
25.1, 23.5; HRMS (CI) Calculated for $\mathrm{C}_{14} \mathrm{H}_{16} \mathrm{~N}_{2} \mathrm{O}_{2}(\mathrm{M}+)$ : 244.1212, found: 244.1218 . 


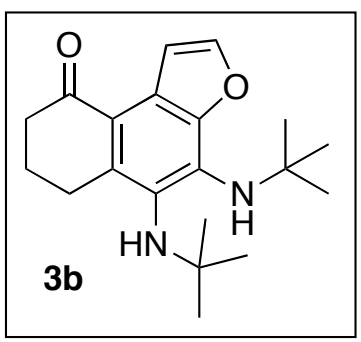

Benzofuran 3b: To a solution of 2-furan-3-yl-cyclohex-2-enone $1(50 \mathrm{mg}, 0.27 \mathrm{mmol})$ in THF $(3 \mathrm{~mL})$ was added molecular sieves and tert-butyl isocyanide ${ }^{4}(77 \mathrm{mg}, 0.10 \mathrm{~mL}, 0.924 \mathrm{mmol})$. After stirring for 15 minutes, the solution was cooled to $0^{\circ} \mathrm{C}$ and diethylaluminum chloride $(0.37 \mathrm{~mL}, 0.37 \mathrm{mmol})$ was added and the reaction was allowed to warm over 18 hours. The reaction mixture was poured into an aqueous $\mathrm{K}_{2} \mathrm{CO}_{3}$ solution ( $200 \mathrm{mg}$ in $10 \mathrm{~mL} \mathrm{H}_{2} \mathrm{O}$ ) and extracted with ethyl acetate several times. The combined organic layers were dried over sodium sulfate, filtered and concentrated. The crude residue was purified by silica gel chromatography (0-3\% EE/DCM) to afford benzofuran $\mathbf{3 b}(59 \mathrm{mg}, 58 \%)$ as pale yellow oil.

$\mathrm{R}_{f}=0.29$ (6\% EE/DCM); IR (film, $\left.\mathrm{cm}^{-1}\right): 3316,2966,2868,1639,1595,1570,1531$, 1386, 1364, 1354, 1290, 1223, 1207, 1183, 1138; ${ }^{1} \mathrm{H}$ NMR (500 MHz, $\left.\mathrm{CDCl}_{3}\right)$ 8: 7.74 (d, $\mathrm{J}=2.0 \mathrm{~Hz}, 1 \mathrm{H}), 7.69(\mathrm{~d}, \mathrm{~J}=2.0 \mathrm{~Hz}, 1 \mathrm{H}), 6.53(\mathrm{br} \mathrm{s}, 1 \mathrm{H}, \mathrm{NH}), 2.86(\mathrm{t}, \mathrm{J}=5.8 \mathrm{~Hz}, 2 \mathrm{H})$, $2.58(\mathrm{t}, \mathrm{J}=6.3 \mathrm{~Hz}, 2 \mathrm{H}), 2.44(\mathrm{br} \mathrm{s}, 1 \mathrm{H}), 2.05$ (quint, J $=6.4 \mathrm{~Hz}, 2 \mathrm{H}), 1.51(\mathrm{~s}, 9 \mathrm{H}), 1.22(\mathrm{~s}$, 9H); ${ }^{13} \mathrm{C}$ NMR (125 MHz, $\mathrm{CDCl}_{3}$ ) $\delta: 196.9,145.9,140.1,139.5,138.2,127.3,124.0$, 113.3, 108.8, 55.0, 51.9, 39.0, 31.1, 30.9, 28.1, 23.7; HRMS (ES) Calculated for $\mathrm{C}_{20} \mathrm{H}_{28} \mathrm{~N}_{2} \mathrm{O}_{2}+\mathrm{Na}: 351.2048$, found: 351.2038 .

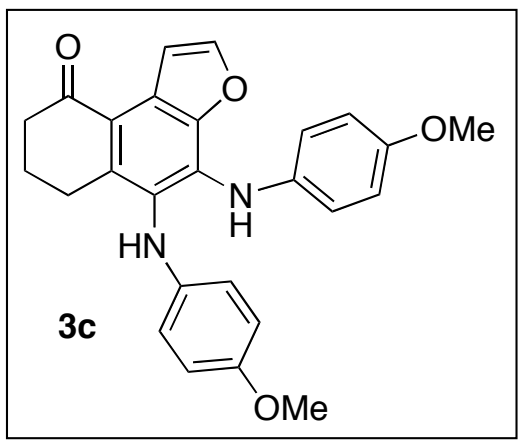

Benzofuran 3c: 2-Furan-3-yl-cyclohex-2-enone 1 (50 $\mathrm{mg}, 0.308 \mathrm{mmol}$ ) and 4-methoxyphenyl isocyanide (123 $\mathrm{mg}, 0.924 \mathrm{mmol}$ ) were azeotroped with benzene three times and further dried under vacuum for 5 minutes. THF ( $3 \mathrm{~mL}$ ) was added and to this solution was added diethylaluminum chloride $(1 \mathrm{M}$ in hexanes, $0.37 \mathrm{~mL}, 0.37 \mathrm{mmol}$ ) at $0{ }^{\circ} \mathrm{C}$. The reaction was allowed to stir at $25{ }^{\circ} \mathrm{C}$ for 18 hours and was poured into aqueous potassium carbonate ( $200 \mathrm{mg}$ in $10 \mathrm{~mL}$ water). The aqueous layer was washed with ethyl acetate several times. The combined organic layers were dried over sodium sulfate, filtered and concentrated. The crude residue was purified by silica gel chromatography $(0-4 \%$ $\mathrm{EE} / \mathrm{DCM})$ to afford compound $3 \mathrm{c}(56 \mathrm{mg}, 43 \%, 46 \% \mathrm{BRSM})$ as yellow foam. $\mathrm{R}_{f}=0.30$ (6\% EE/DCM); IR (film, $\left.\mathrm{cm}^{-1}\right): 3333,2942,1649,1569,1509,1458,1386$, 1330, 1237, 1181, 1135, 1034, 912, 823, 730; ${ }^{1} \mathrm{H}$ NMR $\left(500 \mathrm{MHz}, \mathrm{CDCl}_{3}\right) \delta: 7.69$ (d, J = $2.3 \mathrm{~Hz}, 1 \mathrm{H}), 7.55(\mathrm{~d}, \mathrm{~J}=2.3 \mathrm{~Hz}, 1 \mathrm{H}), 6.97(\mathrm{~m}, 2 \mathrm{H}), 6.81(\mathrm{~m}, 3 \mathrm{H}), 6.75(\mathrm{~m}, 2 \mathrm{H}), 6.54(\mathrm{~m}$, $2 \mathrm{H}), 4.96(\mathrm{~s}, 1 \mathrm{H}, \mathrm{NH}), 3.80(\mathrm{~s}, 3 \mathrm{H}), 3.73(\mathrm{~s}, 3 \mathrm{H}), 2.81(\mathrm{t}, \mathrm{J}=7.0 \mathrm{~Hz}, 2 \mathrm{H}), 2.59(\mathrm{t}, \mathrm{J}=7.1$ $\mathrm{Hz}, 2 \mathrm{H}$ ), 2.04 (app quint, $\mathrm{J}=7.5 \mathrm{~Hz}, 2 \mathrm{H}) ;{ }^{13} \mathrm{C}$ NMR $\left(125 \mathrm{MHz}, \mathrm{CDCl}_{3}\right)$ 8: 197.5, 156.2, 153.1, 146.8, 142.5, 141.3, 139.9, 134.3, 134.1, 127.6, 123.5, 121.9, 116.9, 115.0, 114.5,

\footnotetext{
${ }^{4}$ Howell, B. A.; Trahanovsky, W. S. J. Am. Chem. Soc. 1975, 97(8), 2136-2139.

5 4-Methoxyphenyl isocyanide is commercialy available from Aldrich, but was made instead by converting $p$-anisidine to $N$-(4-methoxyphenyl)formamide (59\%) according to the general procedure in: Deetz, M. J.; Fahey, J . E.; Smith, B. D. J. Phys. Org. Chem. 2001, 14(7), 463-467. $N$-(4-methoxyphenyl)formamide was then converted to 4methoxyphenyl isocyanide using $\mathrm{POCl}_{3}$ and triethylamine (81\% yield).
} 
113.8, 108.4, 55.6, 55.4, 38.9, 25.8, 23.2; HRMS (ES) Calculated for $\mathrm{C}_{26} \mathrm{H}_{24} \mathrm{~N}_{2} \mathrm{O}_{4}+\mathrm{H}$ : 429.1814, found: 429.1806 .

Preparation of compounds $3 \mathrm{~d}$ and $\mathbf{4 d}$ using $\mathbf{E t}_{2} \mathbf{A l C l}$ as the Lewis Acid: 2-Furan-3-ylcyclohex-2-enone 1 (50 $\mathrm{mg}, 0.308 \mathrm{mmol})$ was azeotroped with benzene three times. The enone was taken up in THF ( $3 \mathrm{~mL})$ and 2,6-dimethylphenyl isocyanide ${ }^{6}(121 \mathrm{mg}, 0.925$ mmol) was added. The solution was cooled to $0{ }^{\circ} \mathrm{C}$ and diethylaluminum chloride $(1 \mathrm{M}$ in hexanes, $0.37 \mathrm{~mL}, 0.37 \mathrm{mmol})$. The reaction was warmed to $25^{\circ} \mathrm{C}$ and stirred for 18 hours. Additional diethylaluminum chloride $(2 \times 0.1 \mathrm{~mL})$ was added in a 3- hour interval. It was later realized that the product and the starting material were cospotting on the TLC plate using $50 \% \mathrm{EA} / \mathrm{HX}$ as the eluent, so additional diethyl aluminum chloride may not have been necessary. The reaction mixture was poured into an aqueous solution of potassium carbonate ( $200 \mathrm{mg}$ in $10 \mathrm{~mL}$ water). The aqueous layer was washed with ethyl acetate several times. The combined organic layers were dried over sodium sulfate, filtered and concentrated. The crude residue was purified by silica gel chromatography (100\% DCM then 3-10\% EE/DCM) to afford benzofuran 3d (62 mg, 47\%) as yellow solid and imine $4 \mathbf{d}(13 \mathrm{mg}, 14 \%)$ as a yellow film.

Preparation of Benzofuran 3d using $\mathbf{~ G a C l}_{3}$ as the Lewis Acid: 2-Furan-3-yl-cyclohex2-enone 1 (50 mg, $0.308 \mathrm{mmol})$ was azeotroped with benzene four times. The enone was taken up in toluene $(1 \mathrm{~mL})$ and 2,6-dimethylphenyl isocyanide ${ }^{6}(121 \mathrm{mg}, 0.925 \mathrm{mmol})$ was added. To this mixture was added $\mathrm{GaCl}_{3}$ (Aldrich, $0.5 \mathrm{M}$ in pentane, $0.02 \mathrm{~mL}$, $0.0154 \mathrm{mmol}$ ) and the reaction mixture was heated to $60^{\circ} \mathrm{C}$ for 18 hours. An additional amount of $\mathrm{GaCl}_{3}(0.02 \mathrm{~mL}, 0.0154 \mathrm{mmol})$ was added and the reaction was heated for $60^{\circ} \mathrm{C}$ for an additional 18 hours. The reaction mixture was concentrated and the crude residue was purified by silica gel chromatography $(0-2 \% \mathrm{EE} / \mathrm{DCM})$ to afford benzofuran 3d (100 $\mathrm{mg}, 77 \%)$ as yellow solid.

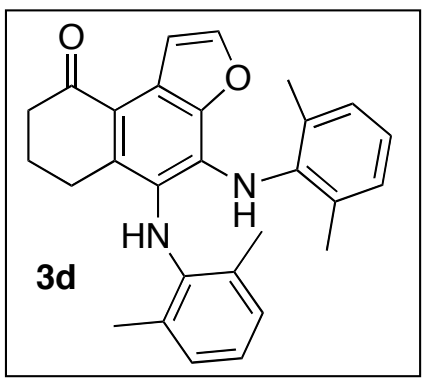

Analytical data for 3d: m.p. $=128-130{ }^{\circ} \mathrm{C} ; \mathrm{R}_{f}=0.42(6 \%$ EE/DCM); IR (film, $\mathrm{cm}^{-1}$ ): 3330, 2945, 1644, 1604, 1567, $1529,1502,1471,1444,1380,1355,1336,1305,1263,1232$, $1185,1135,1098,1031,910,767,731 ;{ }^{1} \mathrm{H}$ NMR $(500 \mathrm{MHz}$, $\left.\mathrm{CDCl}_{3}\right) \delta: 7.61(\mathrm{~d}, \mathrm{~J}=2.0 \mathrm{~Hz}, 1 \mathrm{H}), 7.35(\mathrm{~d}, \mathrm{~J}=2.0 \mathrm{~Hz}, 1 \mathrm{H})$, $7.10(\mathrm{~m}, 3 \mathrm{H}), 7.01(\mathrm{~d}, \mathrm{~J}=7.5 \mathrm{~Hz}, 2 \mathrm{H}), 6.81(\mathrm{t}, \mathrm{J}=7.5 \mathrm{~Hz}$, $1 \mathrm{H}), 6.40(\mathrm{~s}, 1 \mathrm{H}), 4.91(\mathrm{~s}, 1 \mathrm{H}), 2.66(\mathrm{t}, \mathrm{J}=6.0 \mathrm{~Hz}, 2 \mathrm{H}), 2.56$ $(\mathrm{t}, \mathrm{J}=6.1 \mathrm{~Hz}, 2 \mathrm{H}), 2.13(\mathrm{~s}, 6 \mathrm{H}), 2.10(\mathrm{~s}, 6 \mathrm{H}), 2.03$ (app quint, $\mathrm{J}=6.3 \mathrm{~Hz}, 2 \mathrm{H}) ;{ }^{13} \mathrm{C}$ NMR $\left(125 \mathrm{MHz}, \mathrm{CDCl}_{3}\right) \delta: 197.6$, $146.9,142.3,141.4,140.2,138.2,135.6,134.6,129.8,127.9,126.9,126.1,125.1,121.3$, 120.3, 114.6, 108.1, 38.8, 26.4, 23.3, 19.0, 18.5; HRMS (ES) Calculated for $\mathrm{C}_{28} \mathrm{H}_{28} \mathrm{~N}_{2} \mathrm{O}_{2}+\mathrm{H}: 425.2229$, found: 425.2210 .

${ }^{6}$ The isocyanide is commercialy available from Aldrich, but was made instead by converting 2,6-dimethylaniline to $N$-(2,6-dimethylphenyl)formamide according to the procedure in: Deetz, M. J.; Fahey, J . E.; Smith, B. D. J. Phys. Org. Chem. 2001, 14(7), 463-467. $N$-(2,6-dimethylphenyl)formamide was then converted to 2,6-dimethylphenylisocyanide using $\mathrm{POCl}_{3}$ and triethylamine. 


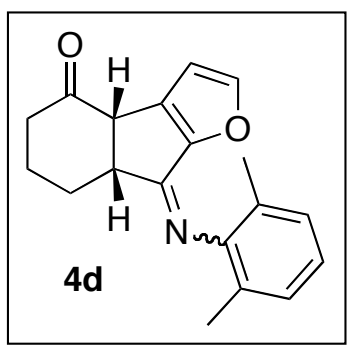

Analytical data for imine 4d: $\mathrm{R}_{f}=0.14(6 \% \mathrm{EE} / \mathrm{DCM})$; IR (film, $\left.\mathrm{cm}^{-1}\right): 2943,1709,1661,1591,1472,1431,1202,1134$, 1092, 899, 765; (note: a small $(\sim 11 \%)$ amount of a geometric isomer is present, so only major isomer is listed in both the proton and carbon data; also, there is atropisomerism present, so each carbon and proton on aryl ring are chemically inequivalent); ${ }^{1} \mathrm{H}$ $\operatorname{NMR}\left(500 \mathrm{MHz}, \mathrm{CDCl}_{3}\right) \delta: 7.39(\mathrm{~d}, \mathrm{~J}=1.8 \mathrm{~Hz}, 1 \mathrm{H}), 7.04(\mathrm{~d}, \mathrm{~J}=$ $7.6 \mathrm{~Hz}, 1 \mathrm{H}), 7.02(\mathrm{~d}, \mathrm{~J}=7.6 \mathrm{~Hz}, 1 \mathrm{H}), 6.95(\mathrm{t}, \mathrm{J}=7.5 \mathrm{~Hz}, 1 \mathrm{H})$, $6.53(\mathrm{~d}, \mathrm{~J}=1.8 \mathrm{~Hz}, 1 \mathrm{H}), 3.94(\operatorname{app~q}, \mathrm{J}=6.7 \mathrm{~Hz}, 1 \mathrm{H}), 3.85(\mathrm{~d}, \mathrm{~J}=7.3 \mathrm{~Hz}, 1 \mathrm{H}), 2.49(\mathrm{~m}$, $2 \mathrm{H}), 2.28(\mathrm{~m}, 2 \mathrm{H}), 2.06(\mathrm{~s}, 3 \mathrm{H}), 2.02(\mathrm{~s}, 3 \mathrm{H}), 1.99(\mathrm{~m}, 2 \mathrm{H}) ;{ }^{13} \mathrm{C}$ NMR $\left(500 \mathrm{MHz}, \mathrm{CDCl}_{3}\right)$

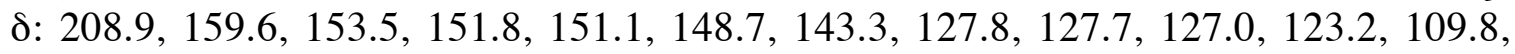
50.8, 46.8, 39.6, 27.8, 20.5, 18.1, 17.9; HRMS (ES) Calculated for $\mathrm{C}_{19} \mathrm{H}_{19} \mathrm{NO}_{2}+\mathrm{H}$ : 294.1494, found: 294.1491 .

Preparation of compounds trans-20 and cis-20: Enone $18^{7}(50 \mathrm{mg}, 0.263 \mathrm{mmol})$ and 2,6-dimethylphenyl isocyanide ${ }^{6}(86 \mathrm{mg}, 0.657 \mathrm{mmol})$ were azeotroped together with benzene three times. The mixture was taken up in THF $(2.6 \mathrm{~mL})$ and cooled to $0{ }^{\circ} \mathrm{C}$. Diethylaluminum chloride $(1 \mathrm{M}$ in hexanes, $0.32 \mathrm{~mL}, 0.32 \mathrm{mmol})$ was added and the reaction was stirred at room temperature for 18 hours. The reaction mixture was poured into aqueous potassium carbonate ( $200 \mathrm{mg}$ in $10 \mathrm{ml}$ in water) and the aqueous layer was extracted with methylene chloride. The combined organic layers were dried over sodium sulfate, filtered and concentrated. The crude ${ }^{1} \mathrm{H}$ NMR indicated 2:1 ratio of products. The residue was purified by silica gel chromatography (5-15\% EA/HX) to give trans-20 (54.5 $\mathrm{mg}), 6.8 \mathrm{mg}$ of a mixture of the two products and the cis-20 $(34.7 \mathrm{mg})$. The trans- and the cis-products were each further purified by preparative thin layer chromatography $(25 \%$ EA/HX) to afford the trans-20 (39 mg) as light orange-yellow solid and the cis-20 (20 $\mathrm{mg}$ ) as yellow crystalline solid (71\% total yield of both isomers). The stereochemistry of both isomers was determined by X-ray crystallographic analysis (see figures 2 and 3 on page 11 and 12 , respectively).

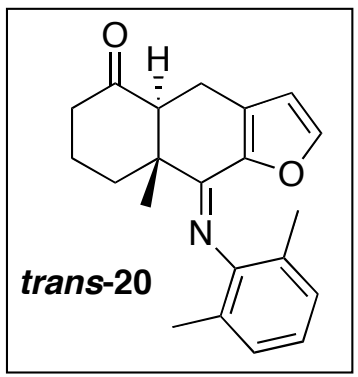

Analytical data for compound trans-20: m.p. $=126-127{ }^{\circ} \mathrm{C} ; \mathrm{R}_{f}$ $=0.38(25 \% \mathrm{EA} / \mathrm{HX})$; IR $\left(\mathrm{film}, \mathrm{cm}^{-1}\right): 2962,2938,1712,1640$, $1592,1467,1432,1373,1269,1216,1185,1161,1090,999,892$, 840, 765, 733; ${ }^{1} \mathrm{H}$ NMR $\left(500 \mathrm{MHz}, \mathrm{CDCl}_{3}\right) \delta: 7.14(\mathrm{~s}, 1 \mathrm{H}), 6.99$ $(\mathrm{m}, 2 \mathrm{H}), 6.87(\mathrm{t}, \mathrm{J}=7.3 \mathrm{~Hz}, 1 \mathrm{H}), 6.25(\mathrm{~s}, 1 \mathrm{H}), 2.95(\mathrm{dd}, \mathrm{J}=4.2$ $\mathrm{Hz}, \mathrm{J}=11.2 \mathrm{~Hz}, 1 \mathrm{H}), 2.89(\mathrm{dd}, \mathrm{J}=11.4 \mathrm{~Hz}, \mathrm{~J}=17.2 \mathrm{~Hz}, 1 \mathrm{H})$, $2.74(\mathrm{dd}, \mathrm{J}=3.7 \mathrm{~Hz}, \mathrm{~J}=17.7 \mathrm{~Hz}, 1 \mathrm{H}), 2.71(\mathrm{~d}, \mathrm{~J}=14.4 \mathrm{~Hz}, 1 \mathrm{H})$, $2.48(\mathrm{~m}, 1 \mathrm{H}), 2.41(\mathrm{ddd}, \mathrm{J}=13.3 \mathrm{~Hz}, \mathrm{~J}=13.3 \mathrm{~Hz}, \mathrm{~J}=6.7 \mathrm{~Hz}$, $1 \mathrm{H}), 2.18(\mathrm{~m}, 1 \mathrm{H}), 2.09(\operatorname{app~dt}, \mathrm{J}=3.4 \mathrm{~Hz}, \mathrm{~J}=14.3 \mathrm{~Hz}, 1 \mathrm{H})$, 2.01-1.94 (m, 7H), $1.19(\mathrm{~s}, 3 \mathrm{H}) ;{ }^{13} \mathrm{C}$ NMR $\left(125 \mathrm{MHz}, \mathrm{CDCl}_{3}\right)$ 8: 210.7, 158.5, 149.4, 145.9, 144.4, 128.6, 127.4, 127.3, 125.5, 124.7, 121.9, 110.7, 55.7, 47.8, 41.0, 34.1, 22.0, 19.9, 19.0, 17.9, 17.8; HRMS (ES) Calculated for $\mathrm{C}_{21} \mathrm{H}_{23} \mathrm{NO}_{2}+\mathrm{H}: 322.1814$, found: 322.1810 .

${ }^{7}$ Chakraborty, A.; Kar, G. K.; Ray, J. K. Tetrahedron 1997, 53, 2989-2996. 


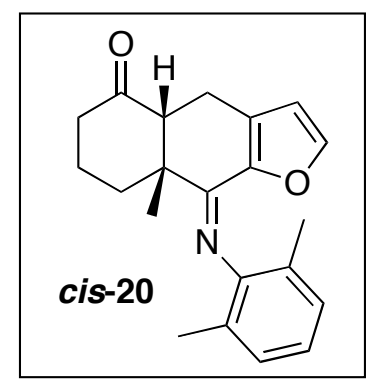

Analytical data for compound cis-20: m.p. $=178-179{ }^{\circ} \mathrm{C} ; \mathrm{R}_{f}=$ 0.26 (25\% EA/HX); IR (film, $\mathrm{cm}^{-1}$ ): 2940, 1712, 1647, 1590, $1462,1435,1374,1349,1324,1264,1204,1161,1091,1035$,

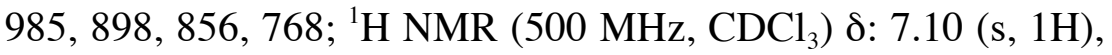
$6.97(\mathrm{~d}, \mathrm{~J}=7.4 \mathrm{~Hz}, 2 \mathrm{H}), 6.86(\mathrm{t}, \mathrm{J}=7.3 \mathrm{~Hz}, 1 \mathrm{H}), 6.27(\mathrm{~s}, 1 \mathrm{H})$, $3.25(\mathrm{~d}, \mathrm{~J}=16.9 \mathrm{~Hz}, 1 \mathrm{H}), 2.97(\mathrm{~d}, \mathrm{~J}=13.5 \mathrm{~Hz}, 1 \mathrm{H}), 2.86(\mathrm{~d}, \mathrm{~J}=$ $4.9 \mathrm{~Hz}, 1 \mathrm{H}), 2.70(\mathrm{dd}, \mathrm{J}=5.2 \mathrm{~Hz}, \mathrm{~J}=16.8 \mathrm{~Hz}, 1 \mathrm{H}), 2.45(\mathrm{~m}, 1 \mathrm{H})$, $2.41(\mathrm{ddd}, \mathrm{J}=6.5 \mathrm{~Hz}, \mathrm{~J}=13.7 \mathrm{~Hz}, \mathrm{~J}=20.2 \mathrm{~Hz}, 1 \mathrm{H}), 2.21$ (ddddd, $\mathrm{J}=3.8 \mathrm{~Hz}, \mathrm{~J}=3.8 \mathrm{~Hz}, \mathrm{~J}=13.0 \mathrm{~Hz}, \mathrm{~J}=13.0 \mathrm{~Hz}, \mathrm{~J}=13.0 \mathrm{~Hz}, 1 \mathrm{H}), 2.03(\mathrm{~m}, 1 \mathrm{H}), 1.94(\mathrm{~s}$, $3 \mathrm{H}), 1.91(\mathrm{~s}, 3 \mathrm{H}), 1.76(\mathrm{ddd}, \mathrm{J}=3.9 \mathrm{~Hz}, \mathrm{~J}=13.1 \mathrm{~Hz}, \mathrm{~J}=13.1 \mathrm{~Hz}, 1 \mathrm{H}), 1.54(\mathrm{~s}, 3 \mathrm{H}) ;{ }^{13} \mathrm{C}$ NMR (125 MHz, $\mathrm{CDCl}_{3}$ ) \&: 209.7, 155.5, 149.7, 146.1, 144.5, 129.7, 127.6, 127.4, 125.3, 125.2, 122.0, 110.7, 56.1, 49.1, 41.2, 34.7, 29.2, 22.8, 19.0, 18.6, 18.0; HRMS (ES) Calculated for $\mathrm{C}_{21} \mathrm{H}_{23} \mathrm{NO}_{2}+\mathrm{H}$ : 322.1807, found: 322.1821 .

Preparation of imine tricycles trans-23 and cis-23: Compound $\mathbf{2 1}^{8}$ (50 $\mathrm{mg}, 0.245$ $\mathrm{mmol}$ ) and 2,6-dimethylphenyl isocyanide ${ }^{6}(80 \mathrm{mg}, 0.612 \mathrm{mmol})$ were azeotroped together with benzene three times. The mixture was taken up in THF $(2.0 \mathrm{~mL})$ and cooled to $0^{\circ} \mathrm{C}$. Diethylaluminum chloride $(1 \mathrm{M}$ in hexanes, $0.29 \mathrm{~mL}, 0.29 \mathrm{mmol})$ was added and the reaction was stirred at room temperature for 48 hours. More isocyanide $(80 \mathrm{mg})$ and diethylaluminum chloride $(0.29 \mathrm{~mL})$ were added and the reaction was allowed to stir an additional 48 hours. The reaction mixture was poured into aqueous potassium carbonate (200 $\mathrm{mg}$ in $10 \mathrm{ml}$ in water) and the aqueous layer was extracted with ethyl acetate. The combined organic layers were dried over sodium sulfate, filtered and concentrated. The crude ${ }^{1} \mathrm{H}$ NMR indicated 1.2:1 ratio of geometric isomers. The crude residue was purified by preparative thin layer chromatography (20\% EA/HX) to give trans-23 (21 mg, 25\%) as pale yellow solid, and an inseparable mixture of starting material and cis-23 (24 mg, $18 \mathrm{mg}$ cis-23 and $6 \mathrm{mg}$ recovered starting material 21 by ${ }^{1} \mathrm{H}$ NMR). The total yield by NMR was $47 \%$. Compound trans-23 was recrystallized from DCM/HX to obtain crystals for X-ray analysis (see figure 4 on page 12).

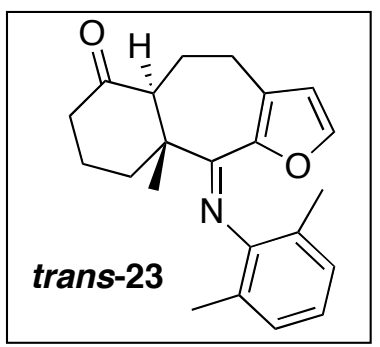

Analytical data for compound trans-23: m.p. $=>175{ }^{\circ} \mathrm{C}$ decomp.; $\mathrm{R}_{f}=0.35$ (25\% EA/HX); IR (film, $\mathrm{cm}^{-1}$ ): 2940, 1712, $1625,1465,1375,1232,1154,1089,1002,761 ;{ }^{1} \mathrm{H}$ NMR $(500$ $\left.\mathrm{MHz}, \mathrm{CDCl}_{3}\right) \delta: 7.01(\mathrm{~d}, \mathrm{~J}=1.4 \mathrm{~Hz}, 1 \mathrm{H}), 6.96(\mathrm{~d}, \mathrm{~J}=7.3 \mathrm{~Hz}$, $1 \mathrm{H}), 6.83(\mathrm{~d}, \mathrm{~J}=7.3 \mathrm{~Hz}, 1 \mathrm{H}), 6.77(\mathrm{t}, \mathrm{J}=7.4 \mathrm{~Hz}, 1 \mathrm{H}), 6.11(\mathrm{~d}, \mathrm{~J}$ $=1.3 \mathrm{~Hz}, 1 \mathrm{H}), 2.80(\mathrm{~d}, \mathrm{~J}=7.6 \mathrm{~Hz}, 1 \mathrm{H}), 2.76(\mathrm{ddd}, \mathrm{J}=2.8 \mathrm{~Hz}, \mathrm{~J}$ $=4.3 \mathrm{~Hz}, \mathrm{~J}=17.5 \mathrm{~Hz}$ ), 2.68 (br m, 1H), 2.51 (ddd, J = 5.5 Hz, J $=12.8 \mathrm{~Hz}, \mathrm{~J}=17.9 \mathrm{~Hz}, 1 \mathrm{H}), 2.46$ (br m, 1H), 2.35 (ddd, $\mathrm{J}=7.5$

$\mathrm{Hz}, \mathrm{J}=\mathrm{J}=13.1 \mathrm{~Hz}, 1 \mathrm{H}), 2.25$ (br m, 1H), 2.20 (ddd, J = 4.7 Hz, J = J = 13.3 Hz, 1H), $2.12(\mathrm{~s}, 3 \mathrm{H}), 2.12-2.07(\mathrm{~m}, 2 \mathrm{H}), 1.75(\mathrm{~s}, 3 \mathrm{H}), 1.60(\mathrm{~m}, 1 \mathrm{H}), 1.33(\mathrm{~s}, 3 \mathrm{H}) ;{ }^{13} \mathrm{C}$ NMR $(125$ $\mathrm{MHz}_{\mathrm{CDCl}}$ ) $\delta: 210.7,164.3,149.5,144.6,144.1,127.2,127.1,126.3,122.9,121.7$, 112.2, 56.5, 52.7, 40.8, 38.5, 25.8, 22.5, 20.0, 19.4, 18.2, 17.7; HRMS (ES) Calculated for $\mathrm{C}_{22} \mathrm{H}_{25} \mathrm{NO}_{2}+\mathrm{H}: 336.1964$, found: 336.1949 .

\footnotetext{
${ }^{8}$ Mal, S. J.; Kar, G. K.; Ray, J. K. Tetrahedron 2004, 60, 2805-2811.
} 


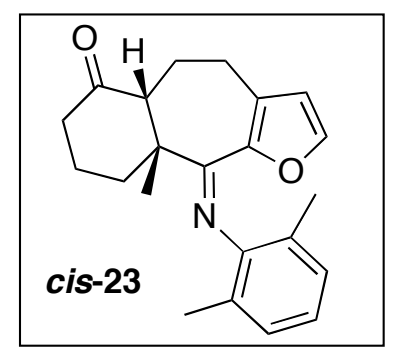

Analytical data for compound cis-23: Compound cis-23 could not be separated from the starting material, but had the following characteristics: $\mathrm{R}_{f}=0.29(25 \% \mathrm{EA} / \mathrm{HX}) ;{ }^{1} \mathrm{H}$ NMR

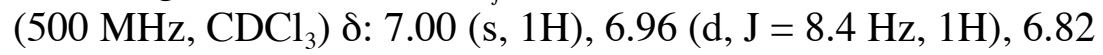
$(\mathrm{d}, \mathrm{J}=8.5 \mathrm{~Hz}, 1 \mathrm{H}), 6.77(\mathrm{dd}, \mathrm{J}=8.6 \mathrm{~Hz}, \mathrm{~J}=8.6 \mathrm{~Hz}, 1 \mathrm{H}), 6.09$ $(\mathrm{s}, 1 \mathrm{H}), 3.60$ (dt, J = 20.4 Hz, J = 4.5 Hz, 1H), 3.39 (ddd, J = 6.4 $\mathrm{Hz}, \mathrm{J}=12.8 \mathrm{~Hz}, \mathrm{~J}=19.7 \mathrm{~Hz}, 1 \mathrm{H}), 2.54-2.31(\mathrm{~m}, 4 \mathrm{H}), 2.25(\mathrm{~m}$, $1 \mathrm{H}), 2.17-2.04(\mathrm{~m}, 2 \mathrm{H}), 2.10(\mathrm{~s}, 3 \mathrm{H}), 1.91(\mathrm{~m}, 2 \mathrm{H}), 1.80(\mathrm{~s}, 3 \mathrm{H})$, 1.36 (s, 3H); HRMS (ES) Calculated for $\mathrm{C}_{22} \mathrm{H}_{25} \mathrm{NO}_{2}+\mathrm{H}$ : 336.1964, found: 336.1952.

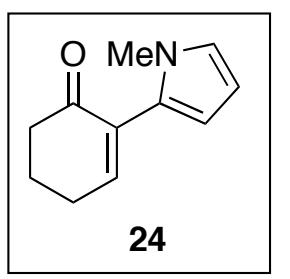

Enone 24: 2-Iodo-cyclohexen-2-one ${ }^{9}$ (500 $\mathrm{mg}, 2.25 \mathrm{mmol}$ ) was azeotroped with benzene three times. The enone was taken up in THF (6 mL) and dichlorobis(triphenylphosphine)-palladium (II) (Aldrich, $79 \mathrm{mg}, 0.113 \mathrm{mmol}$ ) and 1-methyl-2-tributylstannylpyrrole ${ }^{10}$ were $^{-}$ added. The reaction was refluxed for 18 hours. Once cooled to room temperature, the reaction mixture was diluted with diethyl ether and washed with water two times. The organic later was dried over sodium sulfate, filtered and concentrated. Purification by silica gel chromatography afforded compound 24 (98 $\mathrm{mg}, 25 \%$ ) as yellow-brown solid.

m.p. $=47-48{ }^{\circ} \mathrm{C} ; \mathrm{R}_{f}=0.42\left(100 \%\right.$ DCM); IR (film, $\left.\mathrm{cm}^{-1}\right): 2923,2853,1676,1484,1467$, $1426,1410,1352,1304,1252,1152,1121,1089,1054,966,908,814,710 ;{ }^{1} \mathrm{H}$ NMR

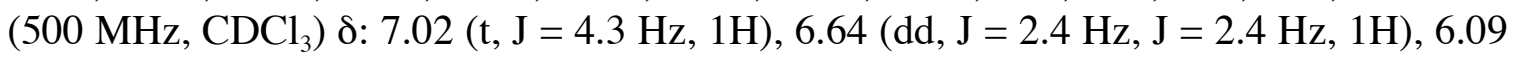
$(\mathrm{dd}, \mathrm{J}=2.8 \mathrm{~Hz}, \mathrm{~J}=3.5 \mathrm{~Hz}, 1 \mathrm{H}), 6.03(\mathrm{dd}, \mathrm{J}=1.8 \mathrm{~Hz}, \mathrm{~J}=3.5 \mathrm{~Hz}, 1 \mathrm{H}), 3.43(\mathrm{~s}, 3 \mathrm{H}), 2.57$ $(\mathrm{m}, 2 \mathrm{H}), 2.52$ (app dt, $\mathrm{J}=4.5, \mathrm{~J}=6.0 \mathrm{~Hz}, 2 \mathrm{H}), 2.11$ (app quintet, $\mathrm{J}=6.1 \mathrm{~Hz}, 2 \mathrm{H}) ;{ }^{13} \mathrm{C}$ NMR $\left(125 \mathrm{MHz}, \mathrm{CDCl}_{3}\right.$ ) $\delta: 197.9,150.4,133.6,129.5,123.2,109.5,107.2,38.8,34.8$, 26.5, 22.8; HRMS Calculated for $\mathrm{C}_{11} \mathrm{H}_{13} \mathrm{NO}^{+}:$175.0997, found, 175.0992 .

Preparation of imine 25 and $\boldsymbol{\alpha}$-hydroxyketone 26: Compound 24 (52 $\mathrm{mg}, 0.297 \mathrm{mmol}$ ) and 2,6-dimethylphenyl isocyanide ${ }^{6}(97 \mathrm{mg}, 0.743 \mathrm{mmol}$ ) were azeotroped together with benzene three times. The mixture was taken up in THF $(3 \mathrm{~mL})$ and diethylaluminum chloride (1M in hexanes, $0.52 \mathrm{~mL}, 0.52 \mathrm{mmol}$ ) was added. Reaction was stirred at room temperature for 18 hours and poured into an aqueous potassium carbonate solution (100 $\mathrm{mg} \mathrm{K}_{2} \mathrm{CO}_{3}$ in $10 \mathrm{~mL} \mathrm{H} \mathrm{H}_{2} \mathrm{O}$ ). The aqueous layer was extracted with methylene chloride several times. The combined ethyl acetate layers were dried over sodium sulfate, filtered, and concentrated. Immediate purification was done by silica gel chromatography (10$50 \% \mathrm{EA} / \mathrm{HX})$ to afford an inseparable mixture of 25 and oxidized product $\mathbf{2 6}$ (5:1 25:26, $53 \mathrm{mg}, \mathbf{5 7 \%}$ yield of mixture) as yellow oil. (Exclusive formation of $\mathbf{2 6}$ occurs if crude mixture is exposed to air for a few hours prior to purification). X-ray data was obtained for $\mathbf{2 6}$ (see figure 5 on page 13).

\footnotetext{
${ }^{9}$ Barriault, L.; Thomas, J. D. O.; Clément, R. J. Org. Chem. 2003, 68, 2317-2323.

${ }^{10}$ (a) Peters, D.; Hörnfeldt, A.-B.; Bronowitz, S. J. Heterocyclic Chem. 1990, 27, 2165.

(b) Bailey, T. Tetrahedron Lett. 1986, 27(37), 4407-4410.
} 

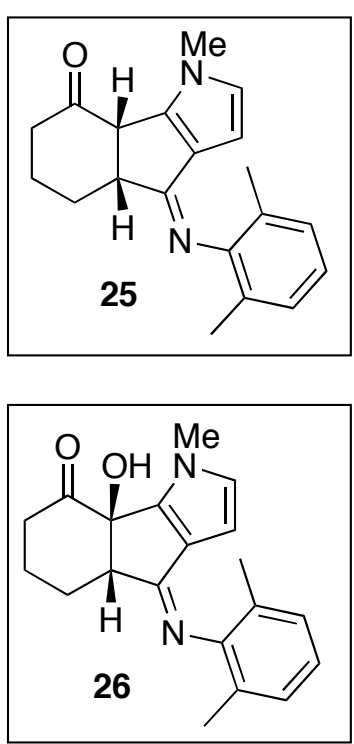

Analytical data for imine 25: $\mathrm{R}_{f}=0.16(50 \% \mathrm{EA} / \mathrm{HX}) ;{ }^{1} \mathrm{H} \mathrm{NMR}$ $\left(500 \mathrm{MHz} \mathrm{CDCl}_{3}\right) \delta: 7.01(\mathrm{~d}, \mathrm{~J}=7.1 \mathrm{~Hz}, 1 \mathrm{H}), 6.99(\mathrm{~d}, \mathrm{~J}=7.3$ $\mathrm{Hz}, 1 \mathrm{H}), 6.90$ (app t, J = 7.4 Hz, 1H), 6.39 (d, J = $2.8 \mathrm{~Hz}, 1 \mathrm{H})$, $4.93(\mathrm{~d}, \mathrm{~J}=2.7 \mathrm{~Hz}, 1 \mathrm{H}), 3.94(\mathrm{~m}, 2 \mathrm{H}), 3.63(\mathrm{~s}, 3 \mathrm{H}), 2.48-2.41(\mathrm{~m}$, $3 \mathrm{H}), 2.16(\mathrm{~m}, 1 \mathrm{H}), 2.04(\mathrm{~s}, 3 \mathrm{H}), 2.01(\mathrm{~s}, 3 \mathrm{H}), 1.98(\mathrm{~m}, 2 \mathrm{H})$; HRMS (ES) Calculated for $\mathrm{C}_{20} \mathrm{H}_{22} \mathrm{~N}_{2} \mathrm{O}+\mathrm{H}: 307.1760$, found: 307.1816.

$\boldsymbol{\alpha}$-Hydroxyketone 26: m.p. $=140-142{ }^{\circ} \mathrm{C} ; \mathrm{R}_{f}=0.38(50 \%$ EA/HX); IR (film, cm ${ }^{-1}$ ): 3349 (br), 2946, 2855, 1712, 1649, 1590, 1518, 1467, 1442, 1414, 1332, 1244, 1204, 1089, 1034, 767, 735; ${ }^{1} \mathrm{H}$ NMR (500 MHz, $\left.\mathrm{CDCl}_{3}\right) \delta: 7.04(\mathrm{~d}, \mathrm{~J}=7.2 \mathrm{~Hz}, 1 \mathrm{H})$, $7.02(\mathrm{~d}, \mathrm{~J}=7.0 \mathrm{~Hz}, 1 \mathrm{H}), 6.93(\operatorname{app~t}, \mathrm{J}=7.4 \mathrm{~Hz}, 1 \mathrm{H}), 6.43(\mathrm{~d}, \mathrm{~J}=$ $2.8 \mathrm{~Hz}, 1 \mathrm{H}), 4.96(\mathrm{~d}, \mathrm{~J}=2.8 \mathrm{~Hz}, 1 \mathrm{H}), 4.52(\mathrm{br} \mathrm{s}, 1 \mathrm{H}), 3.68(\mathrm{~m}$, $1 \mathrm{H}), 3.40(\mathrm{~s}, 3 \mathrm{H}), 2.55-2.51(\mathrm{~m}, 2 \mathrm{H}), 2.46(\mathrm{ddd}, \mathrm{J}=3.2 \mathrm{~Hz}, \mathrm{~J}=$ $9.8 \mathrm{~Hz}, \mathrm{~J}=17.4 \mathrm{~Hz}, 1 \mathrm{H}), 2.19-2.11(\mathrm{~m}, 1 \mathrm{H}), 2.09(\mathrm{~s}, 3 \mathrm{H}), 2.07(\mathrm{~s}$,

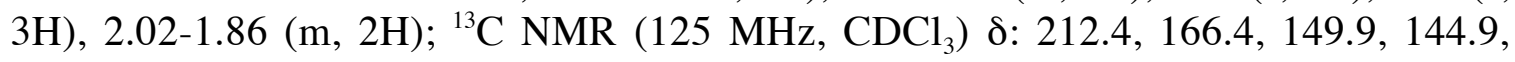
130.4, 128.1, 127.8, 127.5, 127.3, 127.0, 122.9, 103.8, 79.0, 63.2, 34.8, 33.6, 25.5, 20.1, 18.1, 17.9; HRMS (ES) Calculated for $\mathrm{C}_{20} \mathrm{H}_{22} \mathrm{~N}_{2} \mathrm{O}_{2}+\mathrm{H}$ : 323.1760, found: 323.1756.

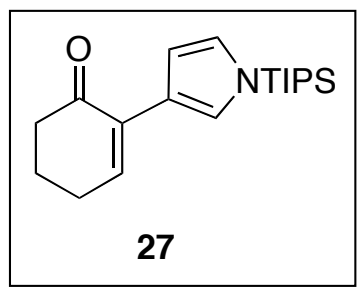

Enone 27: To a solution of 1-(triisopropylsilyl)-3-iodopyrrole ${ }^{11}$ $(1.91 \mathrm{~g}, 5.47 \mathrm{mmol})$ in THF $(32 \mathrm{~mL})$ was added tert-butyl lithium (1.7 $\mathrm{M}$ in pentane, $7.8 \mathrm{~mL}, 13.2 \mathrm{mmol}$ ) at $-78^{\circ} \mathrm{C}$. After stirring for 25 minutes at $-78{ }^{\circ} \mathrm{C}$, zinc (II) chloride (Acros, $0.5 \mathrm{M}$ in THF, $12.6 \mathrm{~mL}, 6.30 \mathrm{mmol}$ ) was added and the reaction was allowed to stir at $0{ }^{\circ} \mathrm{C}$ for 1 hour. The reaction was warmed to room temperature and Argon gas was bubbled through the solution for 15 minutes. Tetrakis(triphenylphosphine)palladium (242 $\mathrm{mg}, 0.21 \mathrm{mmol}$ ) and 2-iodo-cyclohex-2-enone ${ }^{9}(932 \mathrm{mg}, 4.20 \mathrm{mmol}$ ) were added. After stirring for 1.5 hours at room temperature, the reaction was poured into saturated $\mathrm{NH}_{4} \mathrm{Cl}$ and extracted with ethyl ether a few times. The combined ether layers were dried over sodium sulfate, filtered, and concentrated. Purification was achieved by silica gel chromatography (0$10 \% \mathrm{EA} / \mathrm{HX})$ to afford product 27 (345 mg, 26\% yield) as off-white solid.

m.p. $=71-72{ }^{\circ} \mathrm{C} ; \mathrm{R}_{f}=0.61(25 \% \mathrm{EA} / \mathrm{HX}) ; \mathrm{IR}$ (film, $\mathrm{cm}^{-1}$ ): 2945, 2866, 1676, 1616, 1462 ,

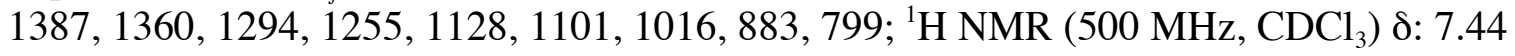
$(\mathrm{s}, 1 \mathrm{H}), 7.11(\mathrm{t}, \mathrm{J}=4.5 \mathrm{~Hz}, 1 \mathrm{H}), 6.72(\mathrm{dd}, \mathrm{J}=\mathrm{J}=2.3 \mathrm{~Hz}, 1 \mathrm{H}), 6.45(\mathrm{~m}, 1 \mathrm{H}), 2.53(\mathrm{t}, \mathrm{J}=$ $6.4 \mathrm{~Hz}, 2 \mathrm{H}), 2.48(\operatorname{app~q}, \mathrm{J}=5.8 \mathrm{~Hz}, 2 \mathrm{H}), 2.03$ (app quint, J = 6.1 Hz, 2H), 1.46 (septet, J $=7.5 \mathrm{~Hz}, 3 \mathrm{H}), 1.10(\mathrm{~d}, \mathrm{~J}=7.5 \mathrm{~Hz}, 18 \mathrm{H}) ;{ }^{13} \mathrm{C} \mathrm{NMR}\left(125 \mathrm{MHz} \mathrm{CDCl}_{3}\right) \delta: 198.8,141.7$, 133.4, 124.4, 123.9, 120.3, 108.2, 39.5, 26.4, 22.9, 17.8, 11.6; HRMS (ES) Calculated for $\mathrm{C}_{19} \mathrm{H}_{31} \mathrm{NOSi}+\mathrm{H}: 318.2253$, found: 318.2242.

\footnotetext{
${ }^{11}$ Alvarez, A.; Guzman, A.; Ruiz, A.; Velarde, E. J. Org. Chem. 1992, 57, 1653-1656.
} 


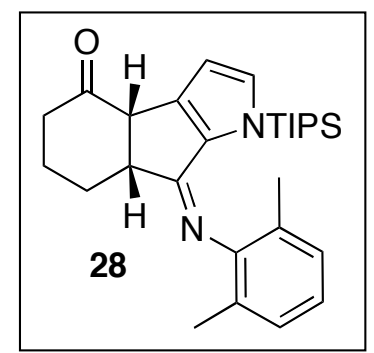

Imine tricycle 28: Enone $27(50 \mathrm{mg}, 0.157 \mathrm{mmol})$ and 2,6dimethylphenylisocyanide ( $31 \mathrm{mg}, 0.236 \mathrm{mmol})$ were azeotroped with benzene three times. THF $(1.6 \mathrm{~mL})$ was added and the solution was cooled to $0{ }^{\circ} \mathrm{C}$. Diethylaluminum chloride $(1 \mathrm{M}$ in hexane, $0.19 \mathrm{~mL}, 0.19 \mathrm{mmol}$ ) was added and the reaction was stirred at room temperature for 8.5 hours. The reaction mixture was poured into aqueous potassium carbonate $(200 \mathrm{mg}$ in $10 \mathrm{ml}$ in water) and the aqueous layer was extracted with ethyl acetate. The combined organic layers were dried over sodium sulfate, filtered, and concentrated. Purification by thin layer preparative chromatography $(40 \% \mathrm{EE} / \mathrm{HX})$ provided compound 3.91 (39 mg, 55\% yield) as a pale yellow oil. NOESY experiment was done to prove cisstereochemistry at the ring-fusion.

$\mathrm{R}_{f}=0.13$ (30\% EE/HX); IR (film, $\mathrm{cm}^{-1}$ ): 2945, 2866, 1710, 1639, 1591, 1466, 1418, 1199, 1136, 1093, 1025, 882, 763; ${ }^{1} \mathrm{H}$ NMR $\left(500 \mathrm{MHz}, \mathrm{CDCl}_{3}\right)$ 8: $7.17(\mathrm{~d}, \mathrm{~J}=2.7 \mathrm{~Hz}$, $1 \mathrm{H}), 7.05(\mathrm{~d}, \mathrm{~J}=7.3 \mathrm{~Hz}, 1 \mathrm{H}), 7.04(\mathrm{~d}, \mathrm{~J}=7.1 \mathrm{~Hz}, 1 \mathrm{H}), 6.89(\operatorname{app~t}, \mathrm{J}=7.4 \mathrm{~Hz}, 1 \mathrm{H}), 6.27$ $(\mathrm{d}, \mathrm{J}=2.6 \mathrm{~Hz}, 1 \mathrm{H}), 3.83(\mathrm{~d}, \mathrm{~J}=6.9 \mathrm{~Hz}, 1 \mathrm{H}), 3.38(\operatorname{app~q}, \mathrm{J}=6.5 \mathrm{~Hz}, 1 \mathrm{H}), 2.33(\mathrm{~m}, 2 \mathrm{H})$, $2.14(\mathrm{~s}, 3 \mathrm{H}), 2.04(\mathrm{~s}, 3 \mathrm{H}), 1.97$ (septet, J = 7.6 Hz, 3H), $1.78(\mathrm{~m}, 1 \mathrm{H}), 1.55(\mathrm{~m}, 2 \mathrm{H}), 1.40$ $(\mathrm{m}, 1 \mathrm{H}), 1.13(\mathrm{~d}, \mathrm{~J}=7.6 \mathrm{~Hz}, 18 \mathrm{H}) ;{ }^{13} \mathrm{C} \mathrm{NMR}\left(125 \mathrm{MHz}, \mathrm{CDCl}_{3}\right) \delta: 210.0,164.5,149.4$, 144.1, 139.2, 136.0, 128.4, 127.7, 124.7, 122.2, 106.8, 49.8, 48.6, 38.6, 26.3, 21.2, 18.7, 18.3, 18.2, 12.9; HRMS (ES) Calculated for $\mathrm{C}_{28} \mathrm{H}_{40} \mathrm{~N}_{2} \mathrm{OSi}+\mathrm{H}$ : 449.2988, found: 449.2977. 


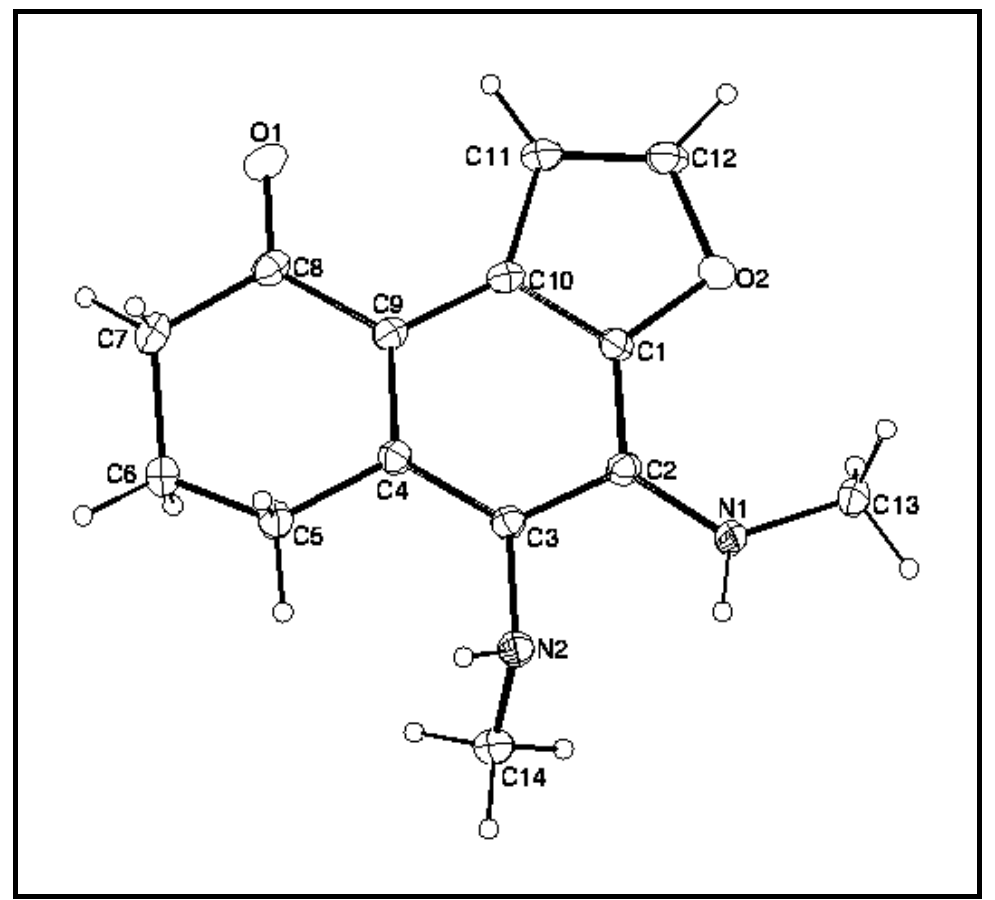

Figure 1. ORTEP drawing of 3a

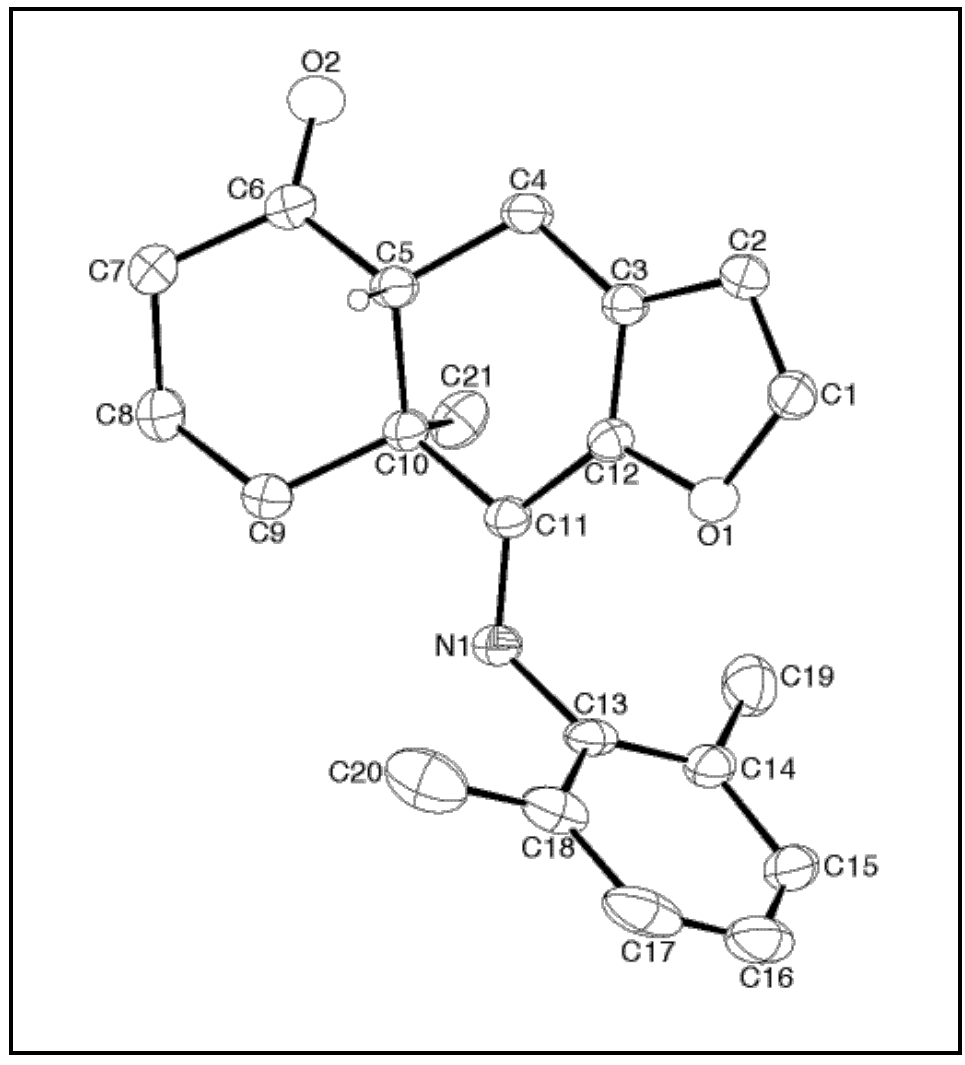

Figure 2. ORTEP drawing of trans-20 


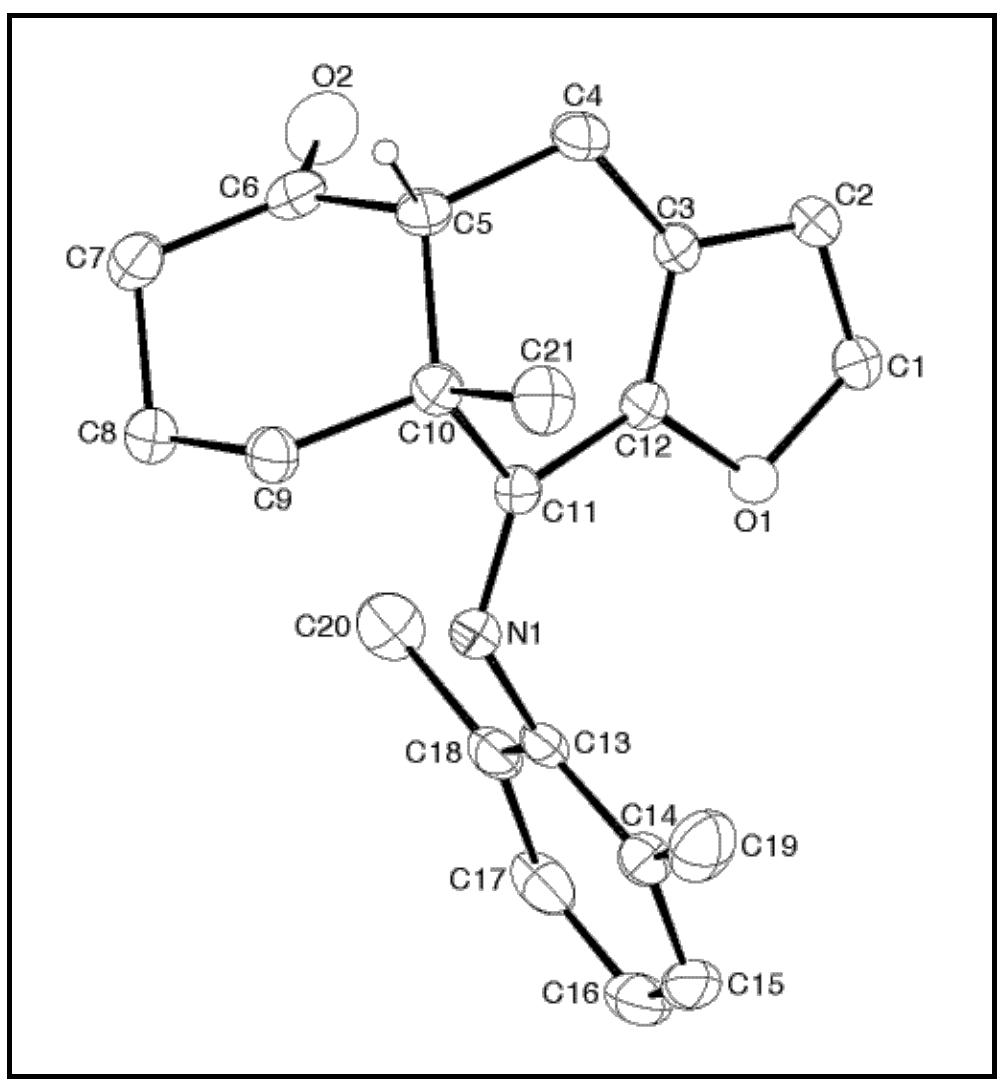

Figure 3. ORTEP drawing of cis-20

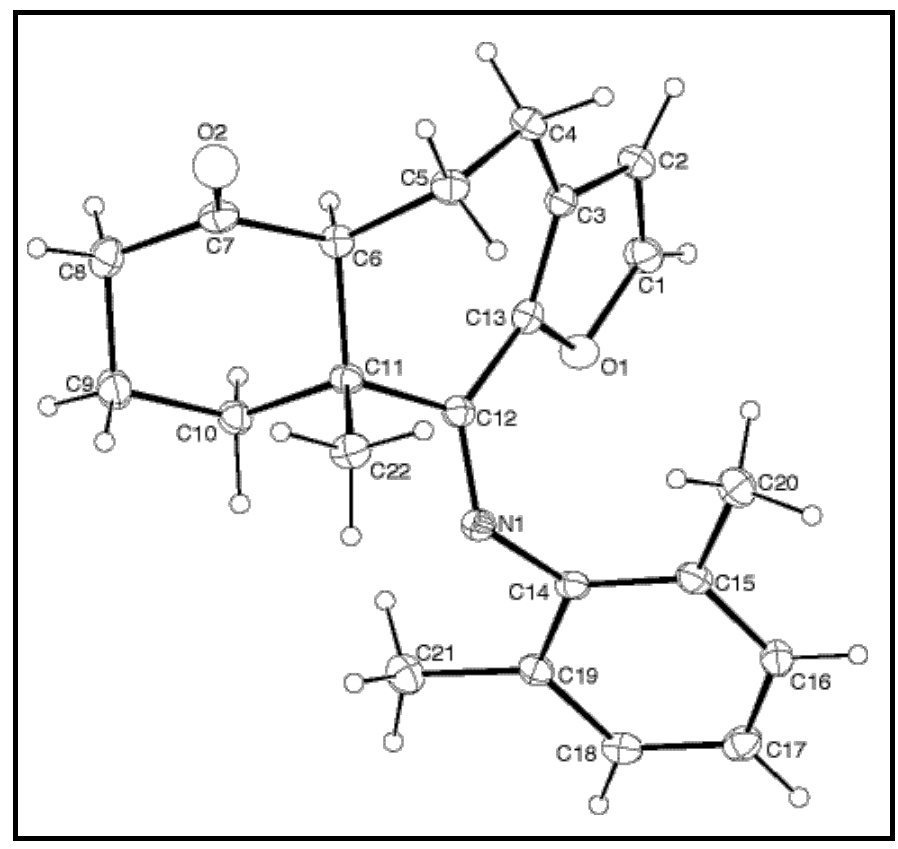

Figure 4. ORTEP drawing of trans-23 


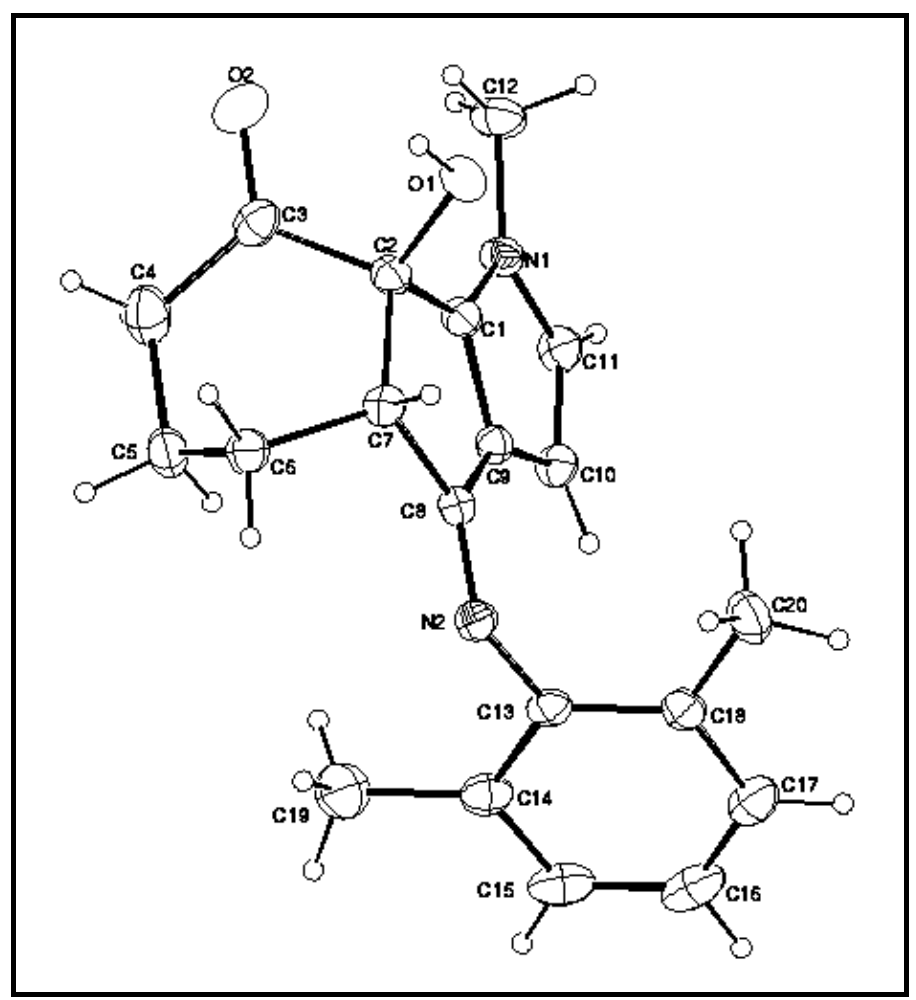

Figure 5. ORTEP drawing of $\mathbf{2 6}$ 